Experi ments on the Excitat $i$ on of the El ectron Waves by a Sl ow Wavegui di ng Ci r cui t

\begin{tabular}{|l|l|}
\hline $\begin{array}{l}\text { j our nal or } \\
\text { publ i cat i on t i t l e }\end{array}$ & 福井大学工学部研究報告 \\
\hline vol une & 27 \\
\hline number & 2 \\
\hline page $r$ ange & $233-237$ \\
\hline year & $1979-09$ \\
\hline URL & ht t p: //hdl . handl e. net /10098/4422 \\
\hline
\end{tabular}


FUKUI UNIVERSITY

VOL. 27 NO. 21979

\section{Experiments on the Excitation of the Electron Waves by a Slow Waveguiding Circuit}

Toshitaka IDEHARA, Haruyuki HAYASHI ${ }^{*}$ and Yoshio ISHIDA

(Received July 3, 1979)

The excitation of the electron plasma wave in a magnetized plasma (Trivelpiece-Gould mode) is investigated experimentally, by using the slow waveguiding circuit (SWC) surrounding the plasma column. The wave vector of the excited wave can be controlled externally.

In a recent few years, the further heating of the magnetically confined plasmas to thermonuclear temperature by using a high power source with a frequency $\omega / 2 \pi$ near the lower hybrid frequency, has been investigated with great interests. For the launched wave to penetrate through the plasma boundany without significant reflection, it is desired that the parallel phase velocity of the wave, $\omega / k_{z}$ (where $k_{z}$ is the wave vector component parallel to the magnetic field $B=B z$ ) is much smaller than the velocity of light $c$. This requirment has been attained by using a finite-length slow waveguiding circuit (SWC).

The propagation of the lower hybrid wave excited by SWC and the mode conversion near the resonance layer were analyzed by several authors. 1),2) on the other hand, it is observed that SWC surrounding the plasma column excites the wave, which propagates in the region of inhomogeneous density distribution and reaches the resonance layer. ${ }^{3), 4)} \quad$ The experimental results can be explained consistently by the theoretical analysis.

This method for the wave excitation has an advantage that the parallel component of the wave vector $\mathrm{k}_{z}$ can be controlled externally and fixed at a suitable value through an experimental study. While, it can not be controlled in the familiar method where the coaxial probe is used for the wave excitation. Considering these points, we will try to apply the former method to the detailed study of the high frequency, electron wave, that is, the Trivelpiece-Gould mode. ${ }^{5)}$ The possibility has been discussed in the previous paper, where the equi-phase lines and damping factor of the excited electron wave are calculated numerically. In this paper, the experimental study of the excitation of electron plasma waves by using SWC is reported.

* Department of Applied Physics 
The experimental apparatus used through the experiments is shown in Fig.l. The plasma is produced by the dc discharge and confined magnetically at the center of the plasma chamber, which consists of the pyrex glass tube of $95 \mathrm{~mm}$ in diameter and $1600 \mathrm{~mm}$ in length. Argon gas is fed continuously through a needle valve and pumped by two 4 inch diffusion pumps, the pressure $p$ at the plasma region being maintained at $1-4 \times 10^{-4}$ Torr. The magnetic field is applied along the tube axis by eight air-core coils, whose intensity distribution is shown in the figure. The plasma parameters are as follows, the plasma frequency $\omega_{\mathrm{p} 0} / 2 \pi$ on the axis of the tube is $1.1-4.3 \times 10^{2} \mathrm{MHz}$ and the electron cyclotron frequency $\omega_{c} / 2 \pi$ is $1.6-5.1 \times 10^{2} \mathrm{MHz}$.

As shown in Fig. 2, SWC which consists of 16 ring electrodes spaced $2 \mathrm{~cm}$ apart, surrounds the glass tube. The wave signal from the signal generator whose frequency $\omega / 2 \pi$ is varried from 25 to $500 \mathrm{MHz}$, is divided into eight signals by three power splitters. They are applied to $S W C$, so that the ring electrodes have their phase alternate by $180^{\circ}$ ( $\pi$-mode). This setup launches a fourwavelength-long axial wave train $\left(2 \pi / k_{z}=4 \mathrm{~cm}\right)$ having $\omega / k_{z}<c$. Waves excited inside the ring structure are detected by a coaxial probe movable axially. By using the interferometer system, the propagating wave patterns along the field (along the axial direction $z$ ) are observed.

Fig. 3 shows typical interferometer traces in the frequency range of $\omega<\omega_{c}$,

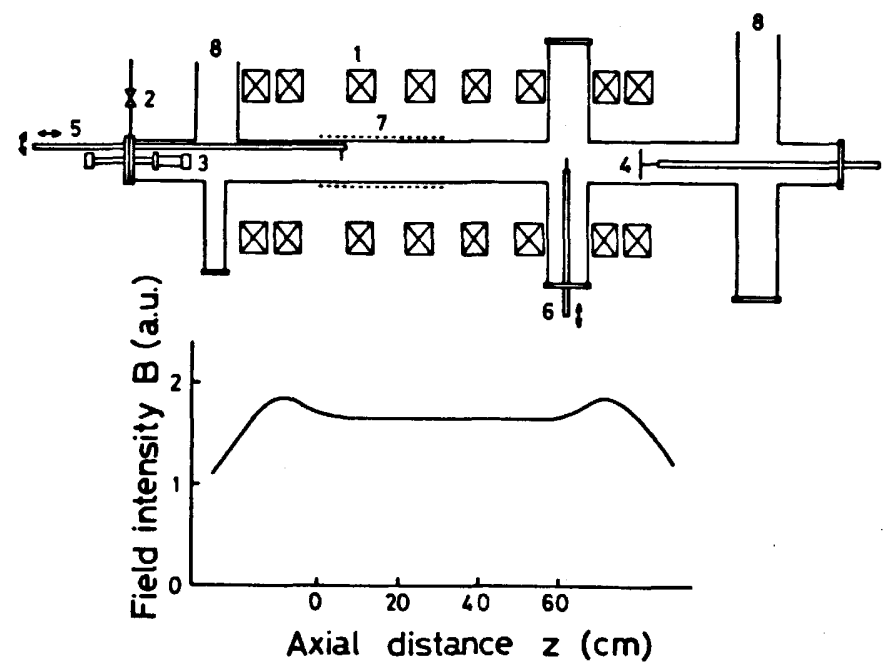

Fig. I The experimental apparatus and the distribution of the applied magnetic field. 1. air-core coils, 2. needle valve, 3. cathode, 4. anode, 5. movable z-probe, 6. movable r-probe, 7. slow waveguiding circuit and 8.4 inch diffusion pumps. 


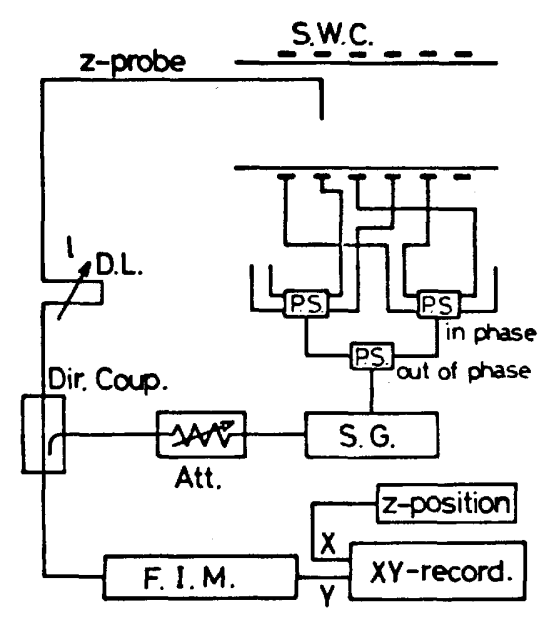

Fig. 2 The block diagram of the measuring system.

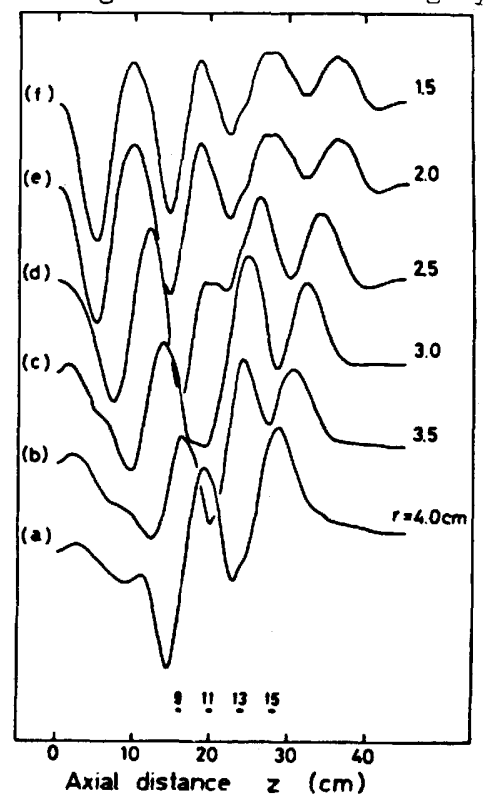

Fig. 3 The propagating wave patterns with the distance $r$ from the axis of the tube as a parameter. The ring electrodes denoted by $9,11,13$ and 15 are used. $\omega / 2 \pi=25 \mathrm{MHz}, \omega_{\mathrm{c}} / 2 \pi=420 \mathrm{MHz}$ and $\omega_{\mathrm{p} 0} / 2 \pi=130 \mathrm{MHz}$. 
with the radial distance $r$ from the axis as a parameter. Here, four rings denoted by $9,11,13$ and 15 are used, so that a two-wavelength-long wave train $\left(2 \pi / \mathrm{k}_{\mathrm{z}}=8 \mathrm{~cm}\right)$ is launched. When the wave propagates in the plasma, the axial position $z$ of the wave packet shifts with the radial distance $r$. Two waves propagating both $+z$ and $-z$ directions are excited in the m-mode operation. The wave number $\mathrm{k}_{\mathrm{z}}$ and the length of a wave train are controlled externally, by the aperture and the number of used ring electrodes.

In Fig. 4, the axial position of the minima (circles) and maxima (triangles) of the observed traces are plotted as functions of the radial distance $r$. Lines connecting the minima (or maxima) show the equi-phase lines. The solid lines show the theoretical results for the equi-phase lines, which are calculated by assuming two dimensional model as follows.

The wave equation can be written as follows,

$$
\left(\frac{\partial}{\partial r}\left(K_{\perp} \frac{\partial}{\partial r}\right)+\frac{\partial}{\partial z}\left(K_{\|} \frac{\partial}{\partial z}\right)\right) \Psi=0,
$$

where

$$
K_{\perp}=1-\frac{\omega_{p}^{2}}{\omega^{2}-\omega_{c}^{2}}, \quad K_{\|}=1-\frac{\omega_{p}^{2}}{\omega^{2}}
$$

and $\omega_{p}$ varries along $r$ and $z$ directions with the plasma density $n(r, z)$.

Actual density profile $\mathrm{n}(\mathrm{r}, \mathrm{z})$ measured by Langmuir probe is used, when eq. (1) is calculated numerically and the equi-phase Iines are determined. The experimental results are fairly good agreement with the theoretical predictions. This fact shows that the electron plasma wave (TrivelpieceGould mode) may be excited by using SWC and, therefore, its wave number $k_{z}$ can be controlled externally.

Fig. 5 shows interferometer traces in the frequency range of $\omega>\omega_{c}$. The axial shift of the wave packet with the radial distance $r$ can not be observed, which means that the standing plasma wave is excited in the frequency range. The detailed study in this case is under way.

\section{References}

1) R.J. Briggs and R.R. Parker, Phys. Rev. Letters 29 (1972) 852.

2) P.M. Bellan and M. Porkolab, Phys. of Fluids 17 (1974) 1592.

3) P.M. Bellan and M. Porkolab, Phys. Rev. Letters 34 (1975) 124.

4) M. Fukushima, Y. Terumichi and S. Tanaka, Kakuyugo Kenkyu 34 (1975) 438. (in Japanese)

5) A.W. Trivelpiece and R.W. Gould, J. Appl. Phys. 30 (1959) 1784.

6) T. Idehara, Jap. J. Appl. Phys. to be published. 


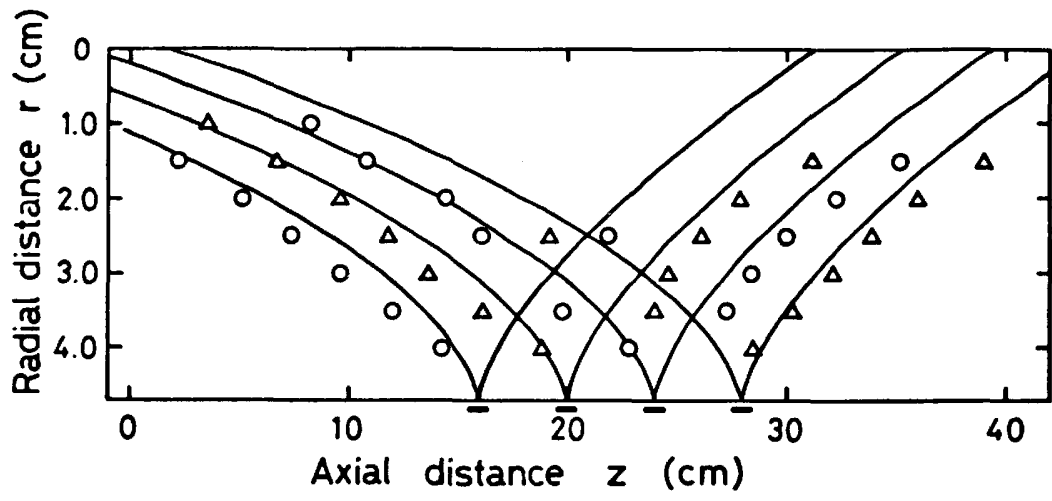

Fig. 4 The equi-phase lines. Circles and triangles show the positions of minima and maxima of wave patterns in Fig. 3. Solid curves are the calculated ones, by using the following values of parameters. $\quad\left(\omega_{c} / \omega_{p}\right)^{2}=10$, $\left(\omega / \omega_{\mathrm{p} 0}\right)^{2}=0.037$ and the angle $\theta$ between the direction of the density gradient and $r$-axis is 0.04 .

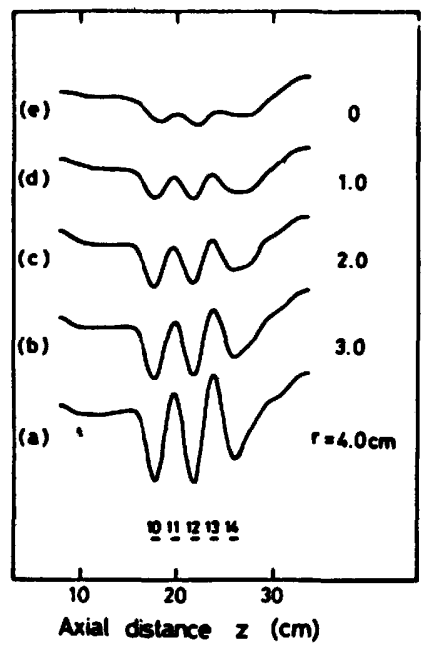

Fig. 5 The propagating wave patterns with the distance $r$ from the axis of the tube as a parameter. $\omega / 2 \pi=500 \mathrm{MHz}$, $\omega_{\mathrm{c}} / 2 \pi=370 \mathrm{MHz}$ and $\omega_{\mathrm{pO}} / 2 \pi=250 \mathrm{MHz}$. 
